\title{
Starspots and photometric noise on observed minus calculated $(\mathrm{O}-\mathrm{C})$ diagrams
}

\author{
A. Kalimeris ${ }^{1}$, H. Rovithis-Livaniou ${ }^{1}$, and P. Rovithis ${ }^{2}$ \\ 1 Sect. of Astrophysics, Astronomy and Mechanics, Dept. of Physics, Univ. of Athens, 15783 Athens, Greece \\ 2 Astronomical Institute, National Observatory of Athens, PO Box 20048, 11810 Athens, Greece
}

Received 14 February 2001 / Accepted 19 March 2002

\begin{abstract}
The effects of starspots on the $\mathrm{O}-\mathrm{C}$ diagrams of eclipsing binaries are investigated, with a special emphasis on contact binaries. It is shown that starspots modulate the $\mathrm{O}-\mathrm{C}$ differences in a fundamentally different way than real orbital period variations. Migrating starspots can only introduce high frequency, low amplitude disturbances (generally less than $0.01 \mathrm{~d}$ ), whose characteristics are numerically specified for typical contact binaries. It becomes evident that the observed systematic short timescale variations of the $\mathrm{O}-\mathrm{C}$ diagrams of active contact binaries (even those like VW Cep) cannot be attributed to starspots. Furthermore, the basic property of non-linear modulation of $\mathrm{O}-\mathrm{C}$ differences is emphasised. This non-linearity has serious consequences for the widespread traditional method of $\mathrm{O}-\mathrm{C}$ diagram analysis. This traditional method is found inappropriate even on a fundamental level, and its mathematical and physical inconsistencies are reviewed.
\end{abstract}

Key words. stars: binaries: eclipsing - starspots - stars: variables: general

\section{Introduction}

Orbital period variations of eclipsing binaries are indirectly observable on their $\mathrm{O}-\mathrm{C}$ diagrams. The $\mathrm{O}-\mathrm{C}$ differences are modulated by all preceding orbital period variations according to the equation (Kalimeris et al. 1994, hereafter K94)

$(\mathrm{O}-\mathrm{C})_{E}=\Delta P(E)+\sum_{k=E_{0}}^{E-2}(E-k) \cdot \Delta P(k+1)$

where $(\mathrm{O}-\mathrm{C})_{E}$ corresponds to the $\mathrm{O}-\mathrm{C}$ difference at the end of the orbital cycle $E, \Delta P(k+1)$ is the variation of the orbital period during the orbital cycle $k+1$, and $E_{0}$ is an initial orbital cycle at which the orbital period is considered known.

Inversely, if the orbital period function $P(E)$ is to be solved from an $\mathrm{O}-\mathrm{C}$ diagram as a function of time, the observed $\mathrm{O}-\mathrm{C}$ differences should be analysed in a way that is consistent with the physical processes occurring in the binary system. Consistency can be ensured only if the function $\Delta T(E)$ that models the observed $\mathrm{O}-\mathrm{C}$ differences, is continuous. If $\Delta T(E)$ reveals discontinuities, the solution for $P(E)$ becomes incompatible with the physics of close binaries. Three techniques have been proposed so far for consistent O-C analysis (K94, Koen 1996;

Send offprint requests to: A. Kalimeris, e-mail: elivan@cc.uoa.gr
Jetsu et al. 1997). In the first method, once a best fitting function $\Delta T(E)$ has been found, the orbital period $P(E)$ can be computed from the simple equation (see K94)

$P(E)=P_{\mathrm{e}}+\Delta T(E)-\Delta T(E-1)$,

where $P_{\mathrm{e}}$ is the adopted ephemeris period.

In the aforementioned way, the orbital period changes of any eclipsing binary can be known for the time interval covered by the $\mathrm{O}-\mathrm{C}$ data (usually from 30 to 100 years). However, the following argument has been raised against the correlation of the observed $\mathrm{O}-\mathrm{C}$ changes with real orbital period variations: photometric disturbances caused by dark starspots can deform a light curve during eclipses. Then, small displacements $\delta \varphi$ in the phases of light minimum are possible. These displacements (that can also be caused by any other asymmetry in the distribution of the photospheric temperature) may produce $\mathrm{O}-\mathrm{C}$ differences that can be falsely interpreted as real orbital period changes. However, the effects of starspots on the O-C differences have never been studied, so that it is rather doubtful whether starspots really are capable of modulating variations in the $\mathrm{O}-\mathrm{C}$ diagrams. The present paper aims to resolve this uncertainty. Since we are primarily interested in the effects of starspots on the $\mathrm{O}-\mathrm{C}$ diagrams of $\mathrm{W} \mathrm{UMa}$ binaries, our study will concentrate on contact systems. An application example concerning W UMa binaries, with characteristics similar to those of $\mathrm{AB}$ And, is given. 


\section{Effects of starspots on the $\mathrm{O}-\mathrm{C}$ diagrams}

Let us assume that $P$ is the orbital period of an eclipsing binary and that the light minimum at the primary eclipse during the orbital cycle $E$ has been shifted by $\delta \varphi$. The epoch of this minimum will be displaced from the proper time $T_{E}$ by

$\delta t=\delta \varphi \cdot P$.

According to Geyer (1977), this $\delta \varphi$ may reach values of about 0.005 . For a binary with an orbital period of about $1 \mathrm{~d}$, such a phase shift will give the impression that an orbital period change equal to $\delta t=\delta \varphi \cdot P \approx 5 \times 10^{-3} \mathrm{~d}$ has occurred.

Consider now a contact binary with a constant orbital period $P_{0}$, having synchronised components $\left(P_{\text {rot }, 1}=\right.$ $\left.P_{\text {rot }, 2}=P_{0}\right)$ and an orbital inclination $i=90^{\circ}$. The position of any spot on the surface of the $j$-component will be denoted by $(\theta, \lambda)_{j}(j=1,2)$, where $\theta$ is the longitude and $\lambda$ is the latitude. On each component, the longitude $\theta$ is measured with respect to the meridian which passes through the centre of the visible disk at the moment of inferior conjunction. This longitude $\theta$ is considered positive when a spot is situated on the leading hemisphere. We further assume that the spots are circular (with an angular radius $r_{\mathrm{S}}$ ), and that the temperature difference with respect to the surrounding photosphere is $\delta T$. Such a spot can cause a phase shift of the light minimum only if the following restrictions hold at the time of conjunction:

a) No other spot with the same physical characteristics $\left(r_{\mathrm{S}}, \delta T\right)$ exists within the positions $(-\theta, \lambda)$ or $(-\theta,-\lambda)$ of the stellar disk;

b) The longitude of the spot is within the interval

$$
90^{\circ}-r_{\mathrm{S}} \leq \theta \leq 90^{\circ}+r_{\mathrm{S}}
$$

If restriction (a) is not satisfied, then two identical spots exist symmetrically with respect to the reference meridian or to the center of the stellar disk. In such a case, one spot cancels out the effects of the other. If restriction (b) is not valid, then the starspot is not visible during the primary eclipse.

\section{1. $\mathrm{O}-\mathrm{C}$ differences due to non-migrating starspots}

Several studies have indicated that starspots on close binaries have angular diameters ranging from $10^{\circ}$ to $40^{\circ}$, which means that they may cover from 5 to $25 \%$ of the whole photosphere of the active components (e.g. Hall \& Busby 1990; Guinan \& Giménez 1993). Although it is not clear whether such dark areas consist of a group of many small spots or of just one giant spot, the second option seems to prevail. In most cases, the active component seems to possess one or two giant spots. It is also argued that such spots can remain on the surface of the active component even for 10 years, or equivalently, over a thousand orbital revolutions. Hence, starspot characteristics are different from those of sunspots, since even at solar cycle maximum

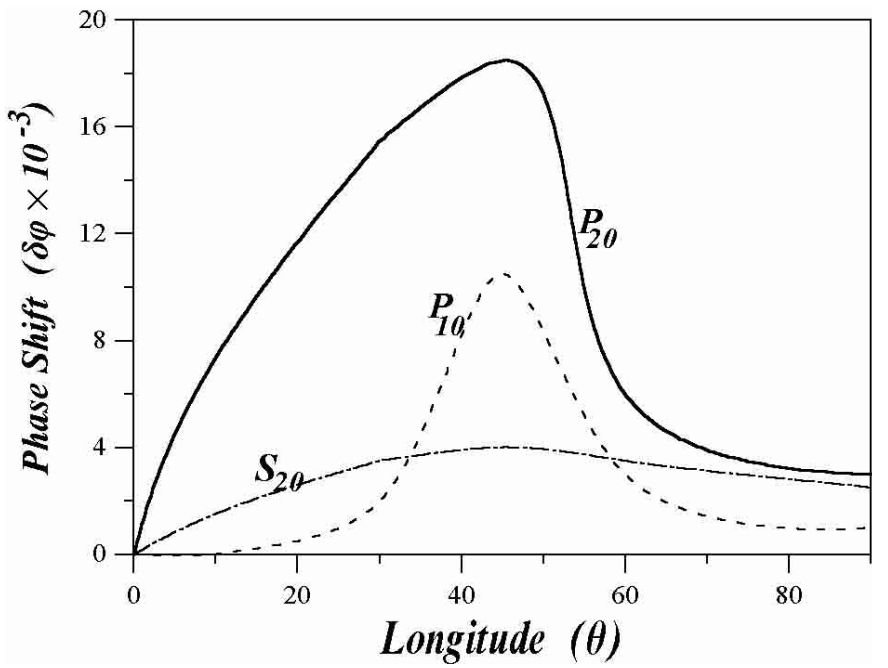

Fig. 1. Phase shift $\delta \varphi(\theta)$ of the primary eclipse of a contact binary similar to AB And. Curves labelled by " $P$ " correspond to a spot on the primary, while " $S$ " refers to a spot on the secondary.

the sunspots rarely cover more than 1 to $2 \%$ of the solar photosphere, and their lifetimes never exceed 3 to 4 solar revolutions.

As for the W UMa type binaries, observations have shown that approximately $20 \%$ to $30 \%$ of all known contact binaries possess one or two giant spots with angular diameters $10^{\circ} \sim 35^{\circ}$ and $\delta T \approx 500 \sim 1500 \mathrm{~K}$ (e.g. Maceroni 1994). Hence, we test the special cases $r_{\mathrm{S}} \approx 20^{\circ}$ and $r_{\mathrm{S}} \approx 10^{\circ}$ with a spot model. We model these two cases for a binary similar to $\mathrm{AB}$ And, and study the resulting $\mathrm{O}-\mathrm{C}$ diagram. The $r_{\mathrm{S}}=20^{\circ}$ case will be referred to as $P_{20}$ when the spot is positioned on the primary component, and as $S_{20}$ when it resides on the secondary. Similarly, a $r_{\mathrm{S}}=10^{\circ}$ spot will be referred to as $P_{10}$ or $S_{10}$. The temperature difference between the dark spot and the surrounding photosphere is fixed to $\delta T=1100 \mathrm{~K}$, which gives $\delta T / T_{\text {eff }} \approx 0.2$ for a system similar to AB And. Since we wish to estimate the maximum effect caused by these spots, it is assumed that they are located on the equator of each component. Using a light curve synthesis code of the Binary Maker type (Bradstreet 1993), but with an enhanced resolution, the phase shift $\delta \varphi$ of the primary minimum has been computed as a function of the spot longitude $\theta$ in the cases $P_{20}, P_{10}, S_{20}$ and $S_{10}$. Results for $\delta \varphi(\theta)$ are shown in Fig. 1 for $0^{\circ} \leq \theta \leq 90^{\circ}$ since $\delta \varphi(\theta)=-\delta \varphi(-\theta)$. Two remarks can be readily made:

i) Spots with the same angular diameters cause significantly greater phase shifts when they lie on the primary component;

ii) Spots on the secondary having $r_{\mathrm{S}}<10^{\circ}$ cause negligible shifts of the light minimum of the primary eclipse (for this reason the curve $S_{10}$ is not shown in Fig. 1).

Conclusion (i) is directly linked to the ratio $r_{2} / r_{1}$ of the radii of equivalent volume. The components of $\mathrm{AB}$ And have $r_{2} / r_{1}=0.72$. Thus a spot with an angular radius $r_{\mathrm{S}}$ 


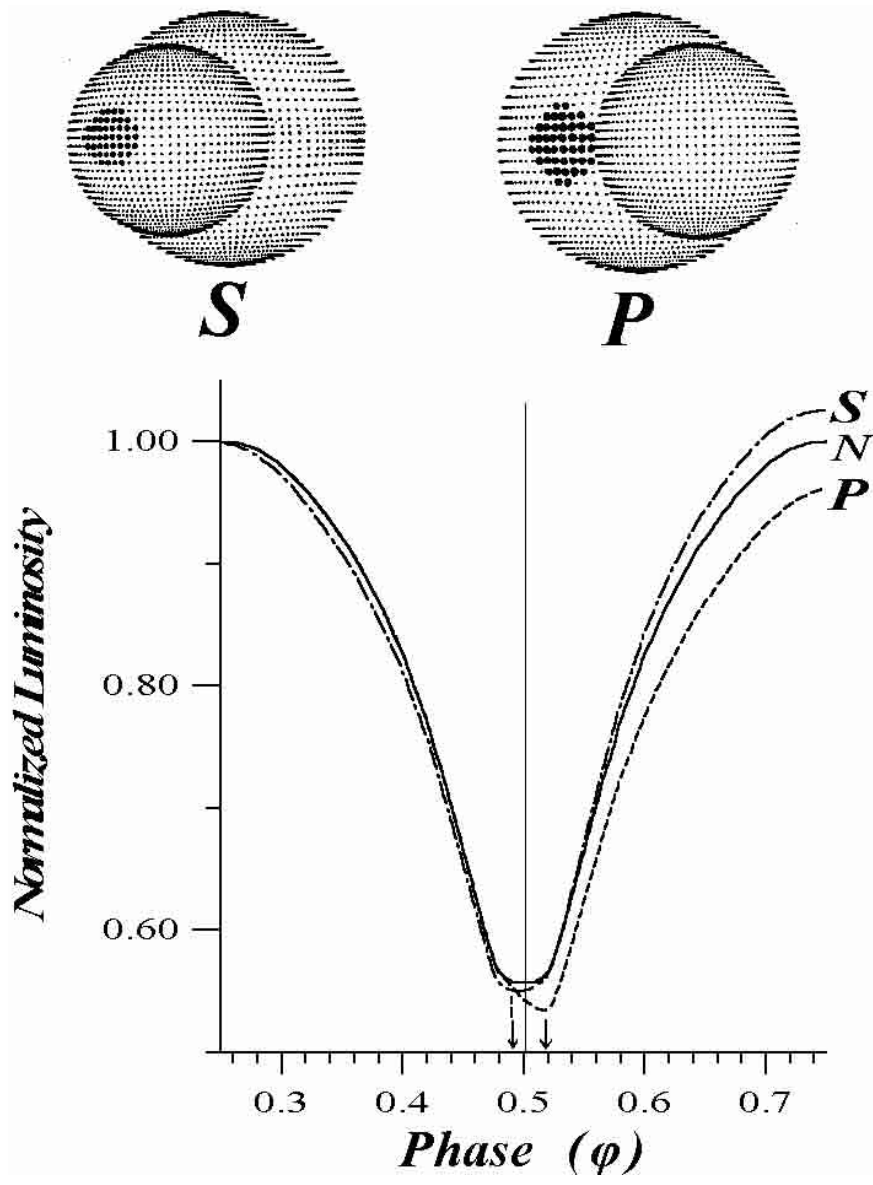

Fig. 2. Light curve distortion and light minimum displacement during primary eclipse, for two particular cases of spots placed on $\mathrm{AB}$ And. These are the $P_{20}$ and the $S_{20}$ spot cases when $\theta=-45^{\circ}$ (marked by $P$ and $S$ respectively). An unspotted light curve (marked by $N$ ) is also given for comparison. Arrows mark the phase of the light minimum.

covers about a two times smaller fractional area of the secondary than that of the primary. Furthermore, the $S$ spots always remain visible during the primary eclipse, while the $P$ spots do not. As a result, the $P$ spots cause a significantly greater photometric perturbation to the light curve than the $S$ spots. This primary eclipse effect, together with other geometrical effects, induces the steep $\delta \varphi(\theta)$ variations for the $P$ spots at $0^{\circ} \leq \theta \leq 60^{\circ}$, as well as the maxima seen between $40^{\circ}$ and $50^{\circ}$. A snapshot, showing in detail the effects of a $S_{20}$ and a $P_{20}$ spot lying at $\theta=-45^{\circ}$, is depicted in Fig. 2. This snapshot reveals that the light minimum precedes the phase 0.5 when the $S_{20}$ spot lies on the trailing hemisphere of the secondary component. Should this $S_{20}$ spot lie on the leading hemisphere of the secondary (i.e. $\theta>0$ ), the phase shift would be opposite, because $\delta \varphi(\theta)=-\delta \varphi(-\theta)$. The opposite behaviour is observed for the $P_{20}$ spot on the primary.

Consider now a sudden appearance of a non-migrating spot. For simplicity, it is assumed that a $P_{20}$ starspot at $\theta=-45^{\circ}$ forms during orbital cycle $E=2$. This starspot distorts the light curve and shifts the primary minimum at the end of the second orbital cycle by $\delta t=\delta \varphi \cdot P_{0}$ in time

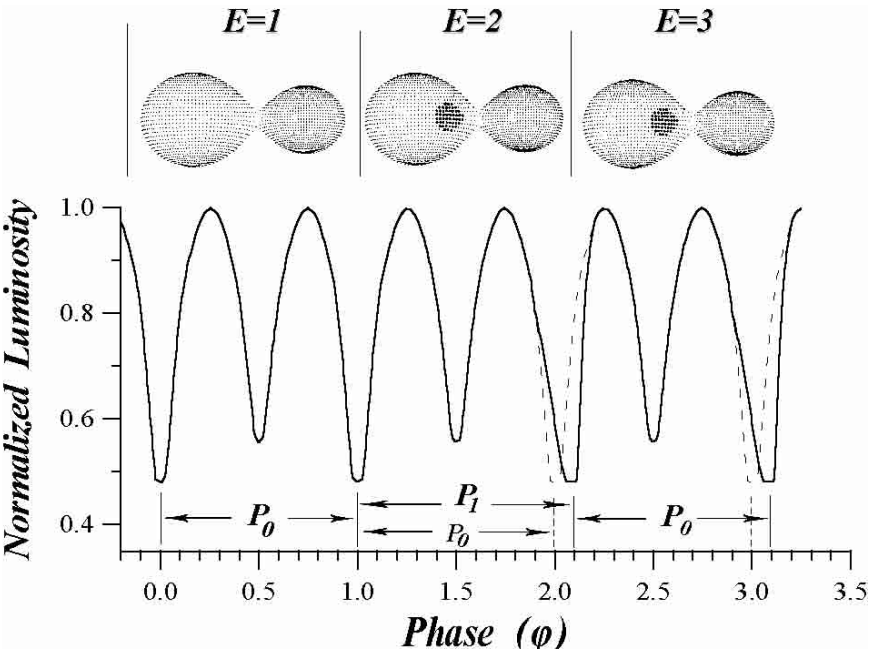

Fig. 3. Three successive light curves of $\mathrm{AB}$ And, (where the phases are measured continuously from 0 to 3 ). A $P_{20}$ starspot forms at $\theta=-45^{\circ}$ during the second orbital cycle. The dashed line represents the shape of the light curves, if the spot would not have formed. The light minimum phases for the spotted and unspotted cases are marked, as well as the intervals between subsequent light minima. A picture of the binary during each orbital cycle is also shown above the light curves.

(where $\delta \varphi>0$, as can be seen from Fig. 1). In this case, the observed duration of the second orbital cycle will be

$P_{1}=P_{0}+\delta t$

This will indicate an apparent (unreal) orbital period change of $\delta P=\delta t$. Since the epoch of primary eclipse at $E$ is generally predicted by the linear ephemeris

$\mathrm{C}_{E}=E_{0}+E \cdot P_{0}$,

then, the epoch of primary light minimum at the end of the second orbital cycle is expected at $\mathrm{C}_{2}=t_{0}+2 \cdot P_{0}$, while the real minimum will be observed at the epoch $\mathrm{O}_{2}=E_{0}+P_{0}+\left(P_{0}+\delta t\right)$. Hence, a difference

$(\mathrm{O}-\mathrm{C})_{2} \equiv \mathrm{O}_{2}-\mathrm{C}_{2}=\left(t_{0}+P_{0}+P_{0}+\delta t\right)$

$-\left(t_{0}+2 \cdot P_{0}\right)=\delta t$

emerges on the $\mathrm{O}-\mathrm{C}$ diagram at $E=2$. A real change $\delta P_{\mathrm{r}}=\delta t$ in the orbital period could cause the same $\mathrm{O}-\mathrm{C}$ difference. Thus, real orbital period changes can not be separated from unreal (i.e. photometric) changes at $E=2$. However, this coincidence does not persist at the subsequent orbital cycles. Indeed, because we suppose that the orbital period of the binary is constant and the starspot did not migrate, the same light curve distortion will be repeated after the next orbital cycle $(E=3)$. Hence the phase shift $\delta \varphi$ at the end of the second, third, and all subsequent cycles will remain the same. Thus, except for $P_{1}$ during $E=2$, the orbital period determined from the light curve will be equal to $P_{0}$ (see also Fig. 3 ). Consequently the corresponding $\mathrm{O}-\mathrm{C}$ difference will be

$(\mathrm{O}-\mathrm{C})_{3} \equiv \mathrm{O}_{3}-\mathrm{C}_{3}=\left(E_{0}+P_{0}+P_{1}+P_{0}\right)$

$-\left(E_{0}+3 \cdot P_{0}\right)=\delta t$. 


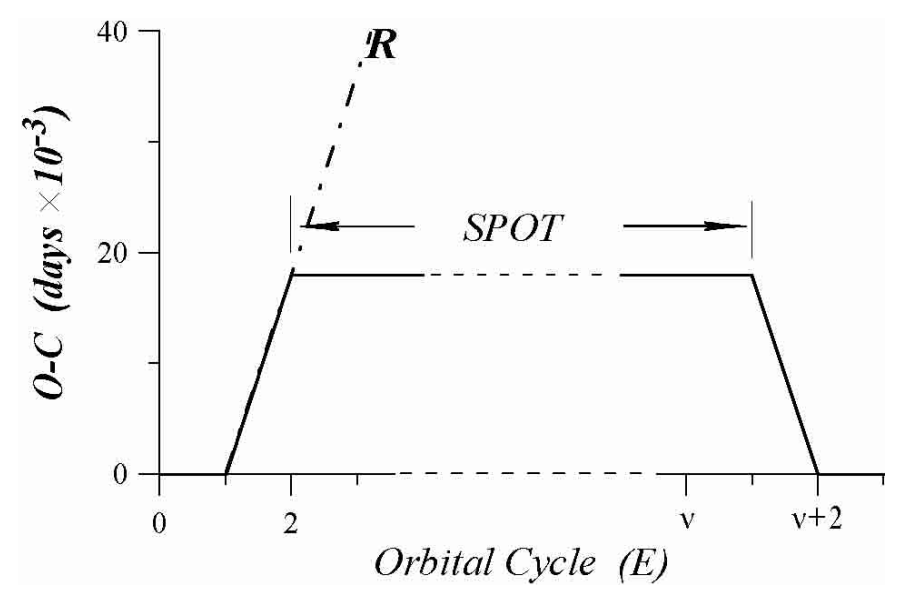

Fig. 4. The O-C diagram due to formation and disappearance of a non-migrating $P_{20}$ spot, which causes an apparent orbital period change of $\delta P$ at $E=2$. If a real orbital period change of $\delta P$ had occurred, the $\mathrm{O}-\mathrm{C}$ diagram would follow the dotteddashed line marked by "R".

This means that the $\mathrm{O}-\mathrm{C}$ difference does not increase after $E=2$, but remains constant and equal to $\delta t=\delta \varphi \cdot P_{0}$, if the starspot does not migrate. On the contrary, if a real orbital period change $\delta P_{\mathrm{r}}=\delta t$ had occurred during the second orbital cycle, the $(\mathrm{O}-\mathrm{C})_{3}$ difference would not have remained constant. In this case, the epoch of the primary eclipse at the end of the third cycle would be

$\mathrm{O}_{3}^{\prime}=E_{0}+P_{0}+\left(P_{0}+\delta P_{\mathrm{r}}\right)+\left(P_{0}+\delta P_{\mathrm{r}}\right) \Rightarrow$

$\mathrm{O}_{3}^{\prime}=E_{0}+3 \cdot P_{0}+2 \cdot \delta t$,

and the respective $\mathrm{O}-\mathrm{C}$ difference would be

$(\mathrm{O}-\mathrm{C})_{3}^{\prime} \equiv \mathrm{O}_{3}^{\prime}-\mathrm{C}_{3}=\left(E_{0}+3 \cdot P_{0}+2 \cdot \delta t\right)$

$-\left(E_{0}+3 \cdot P_{0}\right)=2 \cdot \delta t$.

This result simply verifies the well known fact that a real orbital period change at $E=2$ would cause a linear trend in the $\mathrm{O}-\mathrm{C}$ diagram during all subsequent cycles. Our Fig. 4 illustrates the difference between the $\mathrm{O}-\mathrm{C}$ diagrams caused by a non-migrating starspot, and by a real orbital period change.

If the starspot disappears during the orbital cycle $E=\nu$, the light minimum at the primary eclipse returns to the phase where it was before the spot formed (i.e. 0.0 or 0.5$)$. In other words the effect of the spot on the $\mathrm{O}-\mathrm{C}$ diagram is reversed. Thus, an $\mathrm{O}-\mathrm{C}$ difference of $-\delta t$ occurs at the end of the $\nu+1$ orbital cycle.

The aforementioned analysis illustrates that unlike real orbital period changes, non-migrating starspots cannot cause permanent slopes in the $\mathrm{O}-\mathrm{C}$ diagrams. So far, this fact seems to have eluded detection. However, its consequences in the traditional method of $\mathrm{O}-\mathrm{C}$ diagrams analysis are rather serious (see Sect. 4).

\section{2. $\mathrm{O}-\mathrm{C}$ differences due to migrating starspots}

Due to surface differential rotation, the dark spots migrate in longitude. This generates the well-known migration wave, usually detected in the light curves of RS CVn type of binaries (e.g. Rodonó 1992). Since the position of a starspot on the photosphere of a binary component changes, the value of the phase shift $\delta \varphi$ is changing too. For example, as we showed in Sect. 1, a spot initially appearing at $\theta=-90^{\circ}-r_{\mathrm{S}}$ (i.e. is marginally visible on the limb of the trailing hemisphere during eclipses) and slowly migrating towards positive longitudes, will cause progressively greater phase shifts until it reaches $\theta=-45^{\circ}$. Afterwards, its effect on the primary light minimum will decrease and finally vanish as the spot crosses the meridian $\theta=0^{\circ}$. When the spot enters the leading hemisphere its effects on the light minima will occur in the reverse order. If the starspot survives for a long time, it will appear again on the limb of the trailing hemisphere, and will cause the same $\delta \varphi$ changes that have already been described. In this way, the $\mathrm{O}-\mathrm{C}$ differences caused by long-lived migrating spots can be expected to be periodic.

Consider now a spot migrating on the primary or the secondary component. For simplicity, we assume that the latitude $\lambda$ and the other physical parameters $\left(r_{\mathrm{S}}\right.$ and $\left.\delta T\right)$ remain constant. Also, we assume that the rotation period $P_{\lambda}$ at the latitude $\lambda$ can be approximated by the solar law of surface differential rotation (see e.g. Hall \& Busby 1990)

$P_{\lambda}=P_{\mathrm{eq}} \cdot\left(1-k \cdot \sin ^{2} \lambda\right)^{-1}$,

where $P_{\text {eq }}$ is the equatorial period of rotation (for which $P_{\text {eq }}=P_{0}$ is assumed for each component), and $k$ is the coefficient of differential rotation. The Sun has $k_{\odot}=0.189$, but much lower values have been obtained for the components of close binaries. Observations have indicated a range between $k \approx 6 \times 10^{-4}$ and 0.18 , with a mean value $\bar{k}=3 \times 10^{-2}$ (Hall \& Busby 1990). Thus, the rotation period of a starspot differs from $P_{\text {eq }}$ by $\Delta P=P_{\lambda}-P_{\text {eq }}$, where

$\Delta P=\frac{P_{\mathrm{eq}} \cdot k \cdot \sin ^{2} \lambda}{1-k \cdot \sin ^{2} \lambda}$

At the end of each orbital cycle, a starspot will shift in longitude by

$\delta \theta=2 \pi \frac{\Delta P}{P}$,

where $P$ is the orbital period of the synchronised component. At orbital cycle $E$, the longitude of a spot will be

$\theta_{E}=\theta_{0}+\left(E-E_{0}\right) \cdot \delta \theta$,

where $\theta_{0}$ denotes the longitude where the spot formed on the latitude $\lambda_{0}$ during the orbital cycle $E_{0}$. The appearance of the spot causes a phase shift of the primary minimum, equal to $\delta \varphi\left(\theta_{0}\right)$. One orbital cycle later, the spot's displacement at $\left(\theta_{1}, \lambda_{0}\right)=\left(\theta_{0}+\delta \theta, \lambda_{0}\right)$ induces an additional small phase shift of $\mathrm{d} \varphi\left(\theta_{1}\right)$. Therefore, the total phase shift will be:

$\delta^{\prime} \varphi\left(\theta_{1}\right)=\delta \varphi\left(\theta_{0}\right)+\mathrm{d} \varphi\left(\theta_{1}\right)$.

For a spot lying at $\left(\theta_{1}, \lambda_{0}\right)$, the total phase shift $\delta^{\prime} \varphi\left(\theta_{1}\right)$ should be equal to the value calculated from $\delta \varphi\left(\theta=\theta_{1}\right)$. 
Hence, the equality $\delta^{\prime} \varphi\left(\theta_{1}\right)=\delta \varphi\left(\theta_{1}\right)$ should hold irrespective of whether the spot migrated slowly to $\left(\theta_{1}, \lambda_{0}\right)$ or suddenly formed there. As a result, Eq. (14) gives

$\delta \varphi\left(\theta_{1}\right)=\delta \varphi\left(\theta_{0}\right)+\mathrm{d} \varphi\left(\theta_{1}\right) \Rightarrow$

$\mathrm{d} \varphi\left(\theta_{1}\right)=\delta \varphi\left(\theta_{1}\right)-\delta \varphi\left(\theta_{0}\right)$.

Similarly, a spot migrating from longitude $\theta_{E-1}$ to $\theta_{E}$ one orbital cycle later, will cause a net phase shift equal to

$\mathrm{d} \varphi_{E}=\delta \varphi\left(\theta_{E}\right)-\delta \varphi\left(\theta_{E-1}\right)$.

Then, according to Eq. (3), the apparent orbital period variation within one orbital cycle, will be

$\delta P_{E}=\mathrm{d} \varphi_{E} \cdot P$.

At the orbital cycle $E$, the $\mathrm{O}-\mathrm{C}$ difference obtained from Eqs. (4)-(7) is

$$
\begin{aligned}
& (\mathrm{O}-\mathrm{C})_{E}=\left[t_{0}+P \cdot E+\sum_{i=1}^{E}\left(\delta P_{i}-\delta P_{i-1}\right)\right] \Rightarrow \\
& (\mathrm{O}-\mathrm{C})_{E}=\left[\delta P_{E}-\delta P_{0}\right]=\left[\delta \varphi\left(\theta_{E}\right)-\delta \varphi\left(\theta_{0}\right)\right] \cdot P .
\end{aligned}
$$

Using Eqs. (10)-(18), we computed the O-C diagram for a migrating $P_{20}$ starspot on the primary of a contact binary similar to AB And. Assuming migration rates similar to those found in the very active contact binary VW Cep (where $\Delta P$ is $21 \mathrm{~s}$ at $\lambda \approx 45^{\circ}$ according to Bradstreet $\&$ Guinan 1990), we found that $\Delta P / P \approx 8.7 \times 10^{-4}$, $k \approx 1.7 \times 10^{-3}$, and $\delta \theta=0.31^{\circ} / E$. Since $\Delta P>0$, a spot with the aforementioned migration rate rotates slower than the equatorial region, so it lags by $\delta \theta$ after each orbital cycle. Then, if such a spot appears on the limb of the leading hemisphere, it will migrate across the visible disk of the primary within 575 orbital cycles. Then it will remain out of view for the next 575 orbital cycles. Using the semi-empirical model of Hall \& Busby (1990) for the lifetime $\tau_{\mathrm{S}}$ of giant starspots, and the adopted conditions of our $P_{20}$ spot, we found $770 E<\tau_{\mathrm{S}}<1930 E$. In other words, a migrating $P_{20}$ starspot may complete a few revolutions on the surface of the primary component before fading out. The $\mathrm{O}-\mathrm{C}$ differences caused by such a long-lived spot are shown in Fig. 5. The O-C diagram is periodic when the starspot is visible during eclipses, while the $\mathrm{O}-\mathrm{C}$ differences fall to zero when the spot migrates out of view. The effects of this spot on the $\mathrm{O}-\mathrm{C}$ diagram are largest around $\theta=45^{\circ}$ or $\theta=-45^{\circ}$. If the migration rate of the spot and the orbital period of the binary system remain constant, the $\mathrm{O}-\mathrm{C}$ diagram resembles a wave with an amplitude

$\alpha_{\mathrm{S}}<0.009 \quad$ (days)

and a frequency

$\nu_{\mathrm{S}}=\delta \theta / 2 \pi=\Delta P / P$.

If the orbital period remains constant, this photometric disturbance is always restricted around the zero $\mathrm{O}-\mathrm{C}$ level.

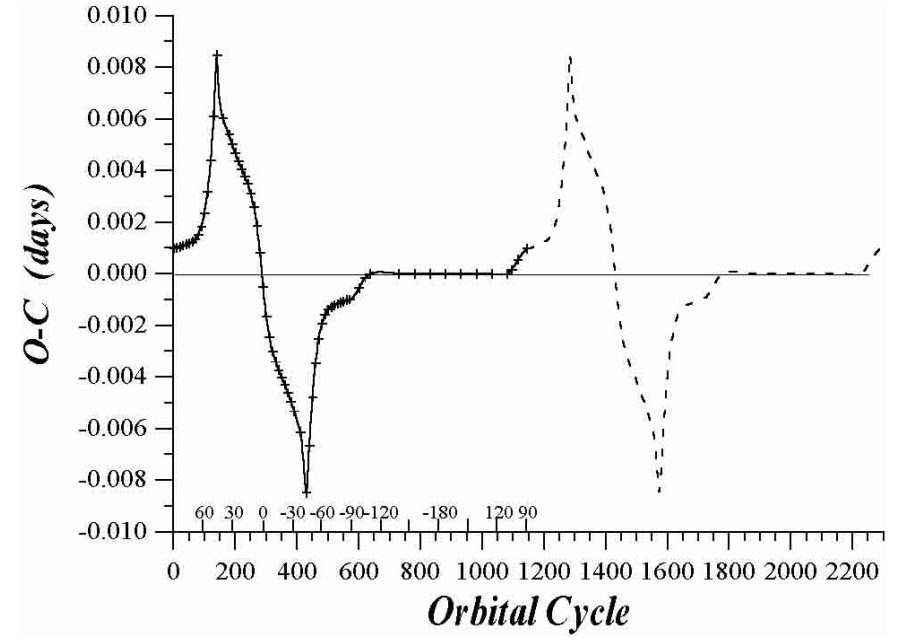

Fig. 5. O-C diagram changes caused by a migrating $P_{20}$ spot on AB And. The diagram covers two complete revolutions of the spot around the photosphere of the primary component. The first revolution is shown by a continuous line, and the second by a dashed line. Small crosses represent the computed values of the $\mathrm{O}-\mathrm{C}$ differences. The longitude $\theta$ of the spot is also marked above the horizontal axis during the first revolution. The $\mathrm{O}-\mathrm{C}$ differences have been computed under the assumption that the real orbital period of the binary remains constant.

If there are real orbital period changes, these disturbances will be superimposed on the real $\mathrm{O}-\mathrm{C}$ changes.

Interesting conclusions can be reached by comparing real $\mathrm{O}-\mathrm{C}$ diagrams with photometric disturbances caused by a $P_{20}$ spot. Two such examples are presented in Figs. 6 and 7 , for $\mathrm{AB}$ And and VW Cep, respectively. If the orbital periods of these binaries were constant, their $\mathrm{O}-\mathrm{C}$ diagrams would show only the photometric disturbances marked by arrows. These disturbances would occur only around the $\mathrm{O}-\mathrm{C}=0$ level. It should be noticed that in general the presence of more than one spot in these binary systems would only decrease the disturbances displayed in Figs. 6 and 7. Thus the well-defined slopes in the observed $\mathrm{O}-\mathrm{C}$ diagrams of both binaries are clearly not caused by photometric disturbances.

The case of VW Cep deserves more attention. This system is one of the most active contact binaries. Its $\mathrm{O}-\mathrm{C}$ diagram has been repeatedly modelled in the traditional way, with a regular quadratic term plus an irregular contribution usually attributed to photometric disturbances (e.g. Kaszás et al. 1998). But our Fig. 7 indicates that even a $P_{20}$ spot could not induce the observed irregularities in the $\mathrm{O}-\mathrm{C}$ diagram of $\mathrm{VW}$ Cep. The $8 \sim 9$ year magnetic activity cycles have been also included (the spot activity is assumed to be present only around the maximum of each magnetic cycle). It is therefore evident that the $\mathrm{O}-\mathrm{C}$ diagram of VW Cep has not been modulated solely by starspots. In other words, the observed shape of the $\mathrm{O}-\mathrm{C}$ diagram betrays the presence of significant real orbital period changes. 


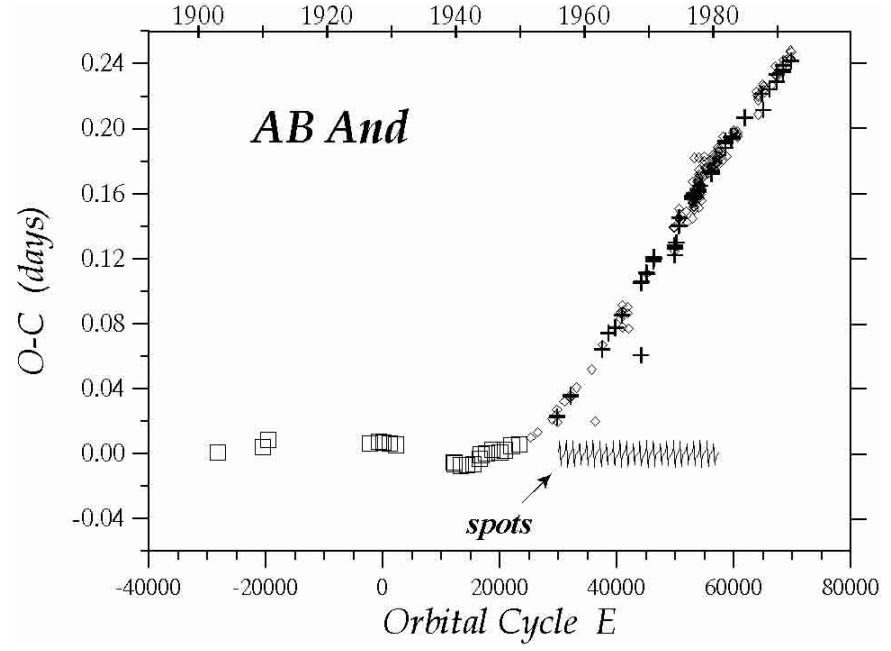

Fig. 6. The observed $\mathrm{O}-\mathrm{C}$ diagram of $\mathrm{AB}$ And and the wave of $\mathrm{O}-\mathrm{C}$ changes that a migrating $P_{20}$ spot could induce. Box symbols on the $\mathrm{O}-\mathrm{C}$ diagram refer to photographic, diamonds to visual, and crosses to photoelectric observations.

\section{Non-linear modulation of the $\mathrm{O}-\mathrm{C}$ diagrams}

Period changes of eclipsing binaries become detectable as slope variations on the $\mathrm{O}-\mathrm{C}$ diagrams. However, an important fact that has been overlooked in the past is that any variation in the slope of an $O-C$ diagram does not only depend on the magnitude $\delta P(E)$ of a period change, but also on the angle $\phi(E)$ between the horizontal axis and the tangent of the $O-C$ diagram at $t=E$. In particular, the greater the local inclination of an $\mathrm{O}-\mathrm{C}$ diagram is, the smaller the slope variation caused by a period change will be. To see this effect, let $\phi_{1}$ and $\phi_{2}$ be the inclinations of the $\mathrm{O}-\mathrm{C}$ diagram before and after $\delta P$ respectively, and $\delta \phi=\phi_{1}-\phi_{2}$. Then,

$\delta P=\tan \phi_{2}-\tan \phi_{1}=\tan \left(\phi_{1}+\delta \phi\right)-\tan \phi_{1}$,

from which, $\delta \phi$ can be expressed as a function of $\phi(E)$ and $\delta P$ by

$\tan \delta \phi=\frac{\delta P}{\delta P \cdot \tan \phi+\tan ^{2} \phi+1}$.

Equation (22) manifests that slope variations of the O-C diagrams are modulated in a non-linear way by $\delta P$. For example, when a unit period change $\delta P_{0}$ happens at a horizontal branch $\left(\phi=0^{\circ}\right)$ of an $\mathrm{O}-\mathrm{C}$ diagram, an inclination change equal to $\tan \delta \phi=\delta P_{0}$ emerges. When $\phi=45^{\circ}$ the same period variation causes a three times smaller inclination change, and when $\phi=60^{\circ}$ the change is six times smaller.

Non-linear modulation of $\mathrm{O}-\mathrm{C}$ diagrams has important consequences for their analysis. In particular, there is a growing risk for any period change to be underestimated or totally undetected, as the slope of an $\mathrm{O}-\mathrm{C}$ diagram increases. In the framework of the traditional method of $\mathrm{O}-\mathrm{C}$ analysis, where period changes are detected by eye inspection through the apparent slope variations they cause, significant orbital events can be misjudged or bypassed.

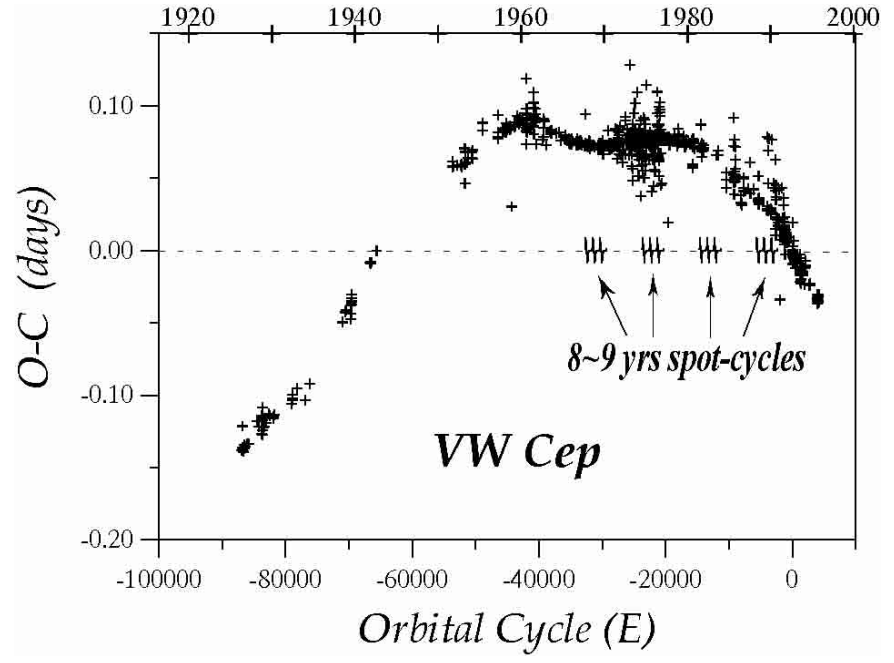

Fig. 7. The same as in Fig. 6, but for the case of VW Cep. Here the spot activity is assumed to present only when the magnetic cycle reaches its maximum.

Because of this fact, the $\mathrm{O}-\mathrm{C}$ modeling becomes ambiguous, since personal judgement plays an essential role, while the orbital history of a binary can be seriously misunderstood. There are many examples in the literature verifying these facts. Among them we mention the cases of AU Ser (Shengbang et al. 1999), of AH Vir (K94), or of SW Cyg (Berrington \& Hall 1994).

Another effect that enhances ambiguity in the traditional analysis is the well-known fact that the shape of an $\mathrm{O}-\mathrm{C}$ diagram depends on the ephemeris elements $\left(E_{0}, P_{0}\right)$ used in the computation of the $\mathrm{O}-\mathrm{C}$ differences. (For example, the shape of the $\mathrm{O}-\mathrm{C}$ diagram of $\mathrm{AB}$ And according to three different ephemerides can be found in Demircan et al. 1994). Hence, an O-C branch that seems to be horizontal in respect to an ephemeris $\left(E_{0}, P_{0}\right)$, may be transformed to a high slope branch when another set of elements $\left(E^{\prime}{ }_{0}, P^{\prime}{ }_{0}\right)$ is used. As a result, an abrupt period change that may be easily detectable at the horizontal branch remains unnoticed on the high slope branch.

\section{Inadequacy of the traditional method of $\mathrm{O}-\mathrm{C}$ analysis}

During the last decades, the great majority of $\mathrm{O}-\mathrm{C}$ diagrams has been analysed with the traditional method. However, serious defects have been detected in this method (e.g. K94). The most significant defects are the violation of uniqueness of the solution for $P(E)$ and contradictions with the physics of close binaries. As a result, an erroneous picture about the real orbital variations of close binaries prevails. The findings in Sects. 2 and 3 add new evidence for the inadequacy of the traditional method, and they also allow us to form an overall picture of its inconsistencies. It will be shown in this section that the traditional method fails due to oversimplifications in its physical assumptions, and due to unnecessary mathematical restrictions. 


\subsection{Normal, irregular, and abrupt changes}

The main restriction in the traditional method emerges from the exclusive use of three specified functions (straight lines, parabolas, or sinusoids) for fitting a time series that shows much more complicated variations. As a result, the physical interpretation of $\mathrm{O}-\mathrm{C}$ diagrams has to be given only in terms of: (a) abrupt period changes (also referred to as period jumps, or sudden changes), (b) period changes at a constant rate, and (c) periodic variations. In this way, the traditional method becomes too deterministic, although the physical phenomenon it deals with (orbital period variations) is not.

Oversimplicity in the traditional method emerges from the fundamental assumption that mass transfer and dynamic phenomena (leading to apsidal motion, light-time effect etc.) modulate the observed period variations. In particular, mass transfer induces parabolic arcs on the $\mathrm{O}-\mathrm{C}$ diagrams, while apsidal motion and light time-effect cause sinusoidal waves. Cycles of intense magnetic activity have also been added to the list of physical mechanisms that may cause periodic variations of the orbital period (Applegate 1992). In this framework, segments of an O-C diagram resembling parabolic arcs or sinusoidal waves are related to a class of orbital changes that are referred to as normal variations. Segments resembling a straight line are also included in normal variations, since they coincide with time intervals of constant orbital period. Variability of any other kind is considered irregular. This classification seems to have been established after the papers of Herczeg (1969), Yamasaki (1975), and Kreiner (1977).

\subsection{Physical and mathematical inconsistencies}

Very often more than one straight line, parabolic arc, or sinusoid, has to be used to fit an $\mathrm{O}-\mathrm{C}$ diagram according to the traditional technique. Then a piecewise approximation is applied, and as a consequence, the fitting functions intersect with each other. These intersections imply discontinuities in the orbital period $P(E)$, which cannot be accepted unless they coincide with epochs of abrupt $\mathrm{O}-\mathrm{C}$ changes. This inevitably drives an observer to search for possible abrupt changes on a $\mathrm{O}-\mathrm{C}$ diagram, at the very beginning of the analysis. But as we showed in Sect. 3, this procedure is ambiguous and reduces the credibility of any $P(E)$ solution.

Once abrupt changes have been identified, the changes seen on the distinct segments (branches) of an $\mathrm{O}-\mathrm{C}$ diagram must be classified either as normal or as irregular. However, irregular variations constitute a serious problem for the traditional method, since they cannot be interpreted via mass transfer or dynamical effects (i.e. the two fundamental physical mechanisms invoked to explain the normal variations). For this reason, they are usually attributed to photometric disturbances (which is an erroneous hypothesis according to the findings of Sect. 2). Alternatively, one may attempt to address the irregular variations in terms of physical mechanisms other than mass transfer or dynamical effects. In this case however, one would be forced to admit orbital variations of whatever kind and rate. Hence, the fundamental distinction between normal and irregular variations would become meaningless and the traditional method would contradict itself.

In order to avoid this contradiction, many analysts apply further segmentation to the irregular parts of the O-C diagrams, until linear or parabolic sections emerge. Doing so, irregular variations are decomposed into numerous normal ones and the procedure is carried on repeatedly, depending on the analyst's personal judgement. This technique is very popular, because the various sections of the divided diagram can be fitted with straight lines, parabolas, or sinusoidal curves, which then offer an obvious advantage for a suitable physical interpretation of the corresponding sections. For example, in the case of SW Cyg (Berrington \& Hall 1994), an apparently smooth O-C diagram has been subdivided into 11 linear segments. But in this way, due to the fitting technique alone, physical events (like the period jumps) that never took place are being introduced. Dozens of similar examples show that the mathematical restrictions used in the traditional method result in a fictitious physical interpretation of the observations.

\section{Conclusions}

In this paper we examined the effects of starspots on the $\mathrm{O}-\mathrm{C}$ differences, and the non-linear modulation of the $\mathrm{O}-\mathrm{C}$ diagrams. Both effects have significant consequences for the traditional method. Our main conclusions are:

i) Starspots cannot cause permanent slopes on the $\mathrm{O}-\mathrm{C}$ diagrams. As a consequence, many $\mathrm{O}-\mathrm{C}$ variations that have been classified as irregular or attributed to photometric disturbances should be reconsidered.

ii) The identification of abrupt changes with the traditional $\mathrm{O}-\mathrm{C}$ diagram analysis is a very uncertain procedure.

iii) The classification of the $\mathrm{O}-\mathrm{C}$ changes into normal and irregular ones is ambiguous.

iv) The results obtained with the traditional method rely on indefinitive concepts (like the abrupt changes), arbitrary procedures (like the decomposition of irregular changes into normal variations), oversimplified physics, personal judgement, and arbitrarily chosen ephemeris elements $\left(E_{0}, P_{0}\right)$. As a result, the $P(E)$ solution is not unique, and the analysis gives a misleading picture about the physical processes acting in close binaries.

Acknowledgements. We gratefully acknowledge the valuable remarks and comments of the referee Dr. L. Jetsu. This research has made use of SIMBAD data base operated at CDS (Strasbourg, France), and NASA's Astrophysics Data System Abstract Service.

\section{References}

Applegate, J. H. 1992, ApJ, 385, 621 
Berrington, R. C., \& Hall, D. S. 1994, AJ, 107, 1868

Bradstreet, D. H. 1993, in Binary Maker User Manual, Contact Software

Bradstreet, D. H., \& Guinan, E. F. 1990, in Active Close Binaries, ed. C. Ibanoglou (Kluwer), NATO ASI Ser., 467

Demircan, O., Derman, E., Akalin, A., et al. 1994, MNRAS, 267,19

Geyer, E. H. 1977, Ap\&SS, 48, 137

Guinan, E. F., \& Giménez, A. 1993, in The realm of interacting binary stars, ed. J. Sahade, G. E. McCluskey, Jr., \& Y. Kondo (Kluwer), 51

Hall, D. S., \& Busby, M. R. 1990, in Active Close Binaries, ed. C. Ibanoglou (Kluwer), NATO ASI Ser., 377

Herczeg, T. 1969, in Mass loss from stars, ed. H. Hack, As. \& Sp. Sc. Lib., 13, 213

Jetsu, L., Pagano, I., Rodono, M., et al. 1997, A\&A, 326, 698

Kalimeris, A., Livaniou-Rovithis, H., \& Rovithis, P. 1994a, A\&A, 282, 775, (K94)
Kaszás, G., Vinko, J., Szatmary, K., et al. 1998, A\&A, 331, 231

Koen, C. 1996, MNRAS, 283, 441

Kreiner, J. M. 1977, in The interaction of variable stars with their envinronment, IAU Col. No. 42, ed. R. Kippenhahn et al.

Maceroni, C. 1994, in Evolutionary links in the zoo of interacting binaries, Proceedings of the Monte Porzio Workshop 1993, ed. F. D'Antona, V. Caloi, C. Maceroni, \& F. Giovannelli, Mem. Soc. As. It., 65-1, 125

Rodonó, M. 1992, in Evolutionary processes in interacting binary stars, ed. Y. Kondo et al. (Kluwer), IAU Symp. 151, 71

Shengbang, Q., Qingyao, L., \& Yulan, Y. 1999, A\&A, 341, 799 Yamasaki, A. 1975, Ap\&SS, 34, 413 